\title{
Accurate Quantitative EDS Mapping at High Count Rates with a Large Area Silicon Drift Detector
}

\author{
C.L. Collins*, J. Holland*, S. R. Burgess*, P.J. Statham*, N. Rowlands** \\ *Oxford Instruments Nanoanalysis, Halifax Road, High Wycombe, HP12 3SE, UK \\ **Oxford Instruments Inc, 300 Baker Avenue, Suite 150, Concord, Massachusetts, USA
}

With the introduction of silicon drift (SDD) detectors, EDS analysis is now possible at acquisition rates in excess of 100,000cps. In particular, rapid qualitative analysis of large areas of a sample is routinely available using spectrum images or X-ray maps.

At high count rates with SDD detectors there is the opportunity for individual photon charge clouds or pulses to overlap causing pile-up problems. Pile-up affects not only the spectrum image but also any spectral data reconstructed from it. Without careful post-collection analysis of the map data or use of specialised pile-up correction software to remove errors [1], these effects can lead to misleading elemental representation errors in the X-ray maps and any spectral data reconstructed from them.

New large area SDDs with active areas up to $80 \mathrm{~mm}^{2}$ have recently emerged which offer the potential for fast analysis with lower beam currents and with improved accuracy. This enables high count rate mapping and quantitative analysis under conditions which have not previously yielded enough count rate. Software pile-up correction can then be used on the collected data to remove residual sum peaks and pile-up continuum from maps and to correct for losses from peaks so that peak area ratios are preserved for accurate quantitative spectral reconstruction. This work follows a previous study which shows that quantitative results from spectrum image data can be as accurate as results from a live spectrum provided accurate livetime correction circuitry is used [2].

Figure 1 shows two maps collected under the same beam current, $\mathrm{kV}$ and geometry conditions on a sample of cast aluminium alloy. Fig 1a shows an EDS map collected using a conventional $10 \mathrm{~mm}^{2}$ SDD detector and Fig. 1b shows data collected with an Oxford Instruments X-Max 80mm ${ }^{2}$ SDD detector. Both maps illustrate the phase differences but it is not until the sum spectra from both maps are compared that the real difference in count rate between the two maps becomes apparent. The map collected with the $80 \mathrm{~mm}^{2}$ detector has over 7 times more counts than the map collected with the $10 \mathrm{~mm}^{2}$ detector. The sum spectra (Fig. 1c) also clearly highlight the problems seen with pile-up in very fast maps. The strong $\mathrm{AlK}_{\alpha}$ signal at $1.48 \mathrm{keV}$ gives a pile up signal at $2.96 \mathrm{keV}$ which in maps can be mistaken for $\mathrm{AgL}_{\alpha}$ if no pile-up correction routines are used. Another pile up peak is seen at $2.4 \mathrm{keV}$ which is a mixture of the $\mathrm{CuL}_{\alpha}+\mathrm{AlK}_{\alpha}$ peaks and could be mistaken for $\mathrm{PbM}_{\alpha}$ at $2.34 \mathrm{keV}$.

Figures $2 \mathrm{a}-\mathrm{c}$ show maps of the elemental distributions of $\mathrm{Al}, \mathrm{Ag}$ and $\mathrm{Pb}$ in the sample. Without investigating each point individually, there is no way to identify whether any of the $\mathrm{Ag}$ or $\mathrm{Pb}$ is actually present in the material. By using a quantitative mapping analysis program which automatically identifies and removes pile-up at each pixel before constructing the map, it is possible to generate quantitative elemental maps which accurately depict the elemental concentrations present in each phase. Figures 2 d-f show these 'Quant Maps'. These maps 
illustrate that the Ag signal is an artefact due to pulse pile up, whereas there are real small precipitates of $\mathrm{Pb}$ present in the sample.

Although mapping at high count rates is becoming more accepted, these results clearly demonstrate the care that must be taken when assessing the validity of results. A combination of pile-up correction, quantitative mapping and a large area SDD detector offers the ability to collect large amounts of data in a very short time and reach realistic conclusions about the specimen.

\section{References}

[1] P.J.Statham, Microchimica Acta, 155, 289-294, 2006

[2] S.R.Burgess et al, Microsc Microanal 13 (Suppl 2), 2007

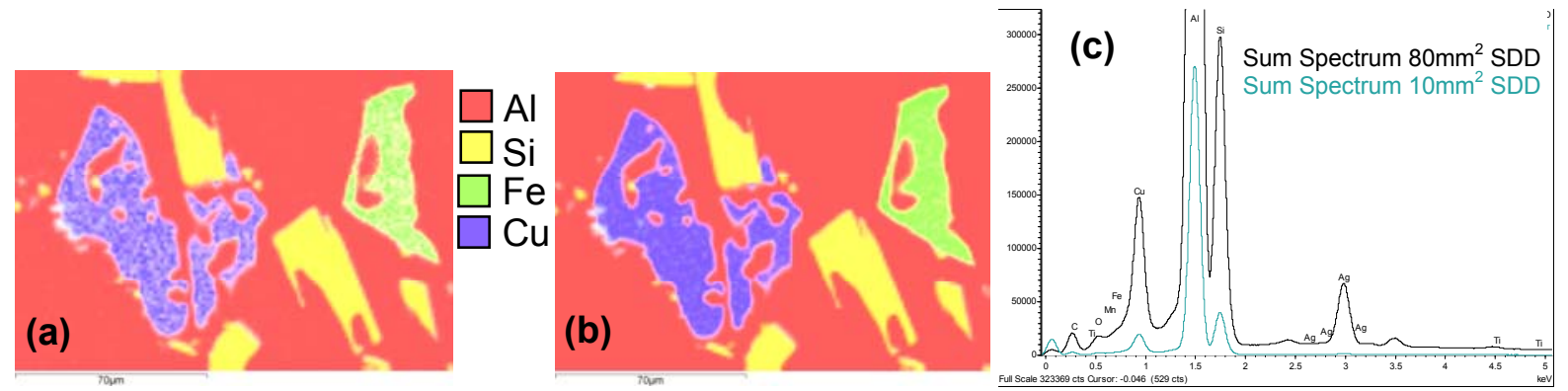

Figure 1. Composite 'mix maps' and associated sum spectrum. (a) Mix map from a conventional $10 \mathrm{~mm}^{2}$ SDD detector showing the aluminium, silicon, iron and copper EDS spectrum images overlaid (b) Mix map from an $80 \mathrm{~mm}^{2}$ large area SDD detector (c) Sum spectra from each of the two mix maps overlaid for comparison.

(a)

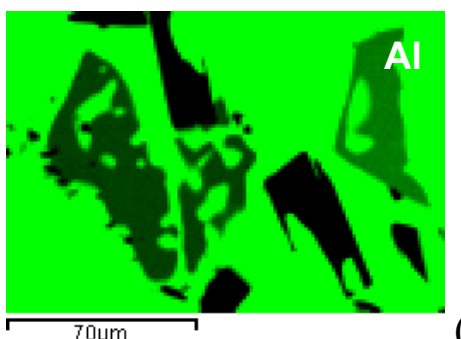

(a)

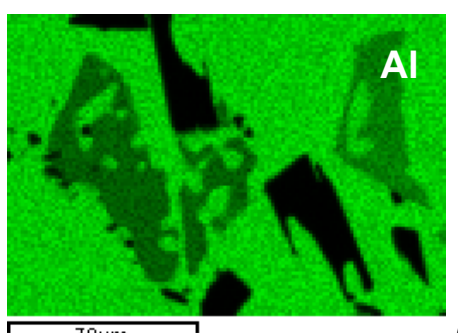

(b)

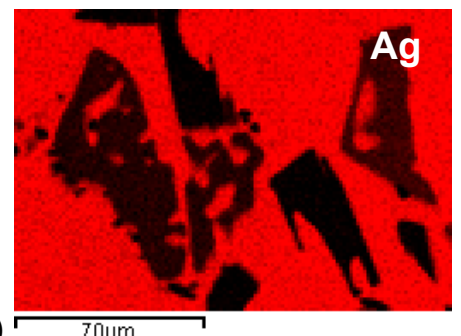

(c)
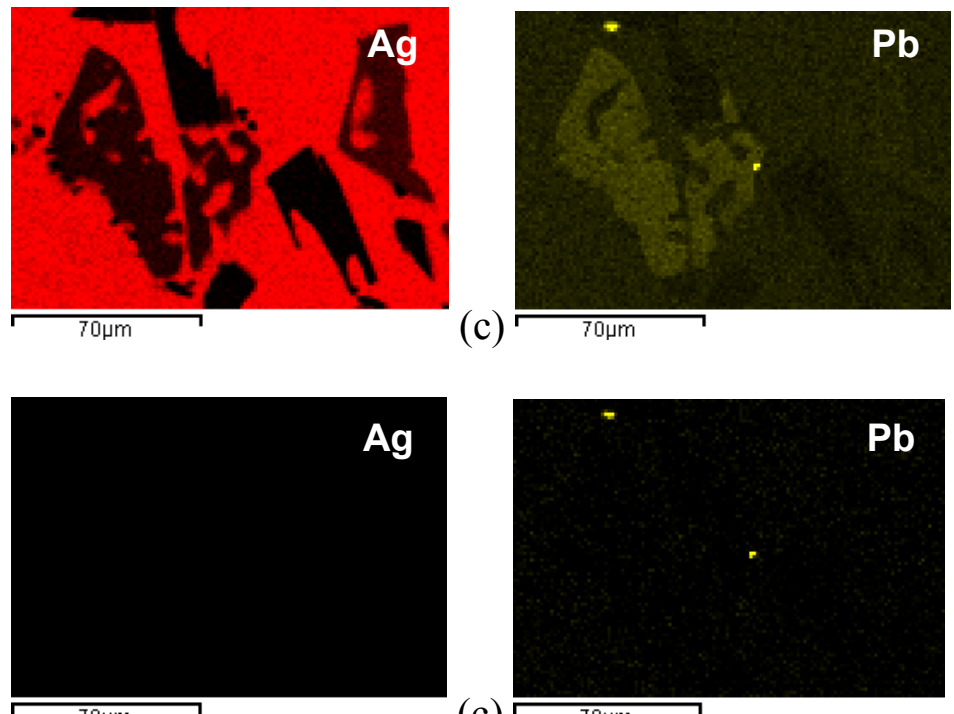

(c)

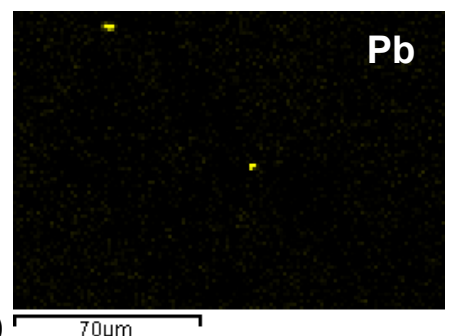

Figure 2. (a-c) Elemental distribution maps displayed without any post-collection analysis. Green $=\mathrm{Al}, \mathrm{Red}=\mathrm{Ag}$ and Yellow $=\mathrm{Pb}$. (d-f) Elemental distribution 'Quant Maps' reconstructed after pile-up correction at each pixel. Green $=\mathrm{Al}, \mathrm{Red}=\mathrm{Ag}$ and Yellow $=\mathrm{Pb}$. 